\title{
Properties of the venous vascular system in the lower extremities of individuals with paraplegia
}

\author{
M T E Hopman MD PhD, ${ }^{1}$ E Nommensen $\mathrm{MS},{ }^{2}$ W N J C van Asten $\mathrm{PhD},{ }^{3}$ \\ B Oeseburg MD PhD, ${ }^{4} \mathrm{R}$ A Binkhorst $\mathrm{PhD}^{4}$ \\ ${ }^{1}$ Assistant Professor of Physiology, ${ }^{2}$ Student Health Science, ${ }^{3}$ Assistant Professor of \\ Vascular Function, ${ }^{4}$ Professor of Physiology, University of Nijmegen, PO Box 9101, \\ $6500 \mathrm{HB}$ Nijmegen, The Netherlands.
}

The purpose of this study was to examine the properties of the venous vascular system in the lower extremities of individuals with long-standing paraplegia (PP). The venous volume variations (VVV), the venous capacity (VC), the venous emptying rate (VER) and the venous flow resistance (VFR) were measured in the left calf of 14 male PP and 12 male able-bodied subjects (ABS) by means of strain-gauge occlusion plethysmography. VVV and VC were significantly lower in PP compared to ABS, $-45 \%$ and $-50 \%$ respectively. Both groups showed a similar resting venous pressure in the calf $(\mathrm{PP}=24.4 \mathrm{mmHg}$; ABS $=19.6 \mathrm{mmHg})$. VER was significantly lower $(-60 \%)$ and hence VFR was significantly higher $(+75 \%)$ in PP compared to ABS. This study demonstrates that the venous vascular properties in the legs of individuals with paraplegia have changed, i.e. a decrease in venous distensibility and capacity and an increase in venous flow resistance. This is most probably the result of vascular adaptations to inactivity and muscle atrophy rather than the effect of a non-working leg-muscle pump and sympathetic denervation.

Keywords: spinal cord injury; strain-gauge plethysmography; venous occlusion plethysmography; venous flow resistance; venous blood pooling.

\section{Introduction}

Confinement to a wheelchair following spinal cord injury (SCI) is not only associated with inactivity and degenerative symptoms such as muscle atrophy and loss of bone integrity but also with changes in the cardiovascular system. Previous studies have been focused on central cardiovascular responses at rest and during exercise in individuals with a SCI. ${ }^{1.2}$ From these studies it is known that cardiac performance has been changed in these individuals. Cardiac performance, which may be determined by the interaction between central venous pressure and cardiac output, is to a large extent influenced by the peripheral circulation through active and passive redistribution of blood.$^{3,4}$ To date, only a few studies have been directed towards the peripheral circulation in individuals with SCI. Previous investigators have examined the vascular responses in the legs of SCI individuals during arm exercise. ${ }^{5-7}$ They reported different responses between SCI and ablebodied subjects (ABS): a clear leg-volume decrease in ABS during arm exercise and no changes $^{5,7}$ or a leg-volume increase ${ }^{6}$ in SCI individuals during arm exercise. These differences between SCI and ABS were attributed to a lack of sympathetic venoconstriction and the inability to activate the muscle pump in the legs in the SCI group. It has been hypothesised that these impairments in SCI may lead to a so called 'venous blood pooling' below the lesion, which would mean a dilated venous vascular system. On the other hand a remarkable muscle atrophy can be observed below the lesion. This may cause the vascular system to adapt to the inactivity and muscle atrophy resulting in a smaller venous vascular volume. This contradiction has never been 
explored and is, therefore, the subject of the underlying study.

The purpose of this study was to examine the properties of the venous vascular system, as characterised by venous volume variation, venous capacity, venous emptying rate and venous flow resistance, in the lower extremities of individuals with paraplegia in comparison to able-bodied control subjects. The results of this study may elucidate the contradiction of 'venous blood pooling' versus 'venous vascular atrophy'.

\section{Methods}

\section{Subjects}

Fourteen male individuals with paraplegia (PP) and 12 male able-bodied control subjects (ABS), aged between 20 and 40 years, participated in this study. The study was approved by the Faculty Ethics Committee and all subjects gave their written informed consent. Seven subjects had complete spinal cord lesions at levels between T4 and T11, whereas the other seven subjects had incomplete lesions at levels between $\mathrm{C} 8$ and $\mathrm{T} 12$. The level as well as the completeness of the lesions were verified by sensor-motor neurological examination. ${ }^{8}$ The lesions had existed between 3 and 11 years and were all of traumatic origin. All subjects underwent a comprehensive medical examination from which no impediment for participation in this study was found. None of the subjects used medicines likely to affect the results. PP and ABS were matched on age, body mass, height and physical fitness (Table I).

\section{Protocol and measurements}

For at least two hours prior to the test subjects refrained from caffeine, nicotine and alcohol. All subjects were weighed on a hospital scale, while body height was taken from the medical file. Calf and thigh circumference were measured, using a measuring tape, at the thickest part while the muscles were relaxed. During the tests the temperature in the experimental room was kept between 21 and $23^{\circ} \mathrm{C}$. PP subjects sat in their own wheelchair and ABS subjects in a chair in the same upright position. The legs were slightly bent, the feet rested on a foot-board, and the heels padded to achieve maximum leg-muscle relaxation. The footboard was adjusted to a height that resulted in a blood column of $35 \mathrm{~cm}$ between the level of the apex of the heart and the measuring point of the strain gauges at the calf, in order to keep the hydrostatic pressure the same for all subjects.

Venous occlusion plethysmography by means of strain gauges, a well-established non-invasive method, was used to examine the properties of the vascular system in the legs. ${ }^{9-11}$ The strain-gauge plethysmograph consisted of two mercury-filled tubes of silicon rubber, which were stretched around the largest girth of the left calf. The relative changes in the calf volume were equal to the relative changes in resistance of the mercury conductor measured as changes in voltage. For calibration of the plethysmograph the electrical method described by Brakkee and Vendrik was used. ${ }^{12}$

A pneumatic cuff of $12 \mathrm{~cm}$ width and $59 \mathrm{~cm}$ in length was put around the left thigh

Table I Physical characteristics of individuals with paraplegia (PP) and able-bodied control subjects $(\mathrm{ABS})$ as mean $\pm \mathrm{SD}$

\begin{tabular}{lrr}
\hline & PP & \multicolumn{1}{c}{ ABS } \\
\hline Age $(\mathrm{yr})$ & $32 \pm 5$ & $31 \pm 6$ \\
Body mass $(\mathrm{kg})$ & $81 \pm 17$ & $78 \pm 11$ \\
Height $(\mathrm{cm})$ & $187 \pm 7$ & $179 \pm 8$ \\
Calf circumference $(\mathrm{cm})$ & $31 \pm 5$ & $38 \pm 2^{*}$ \\
Thigh circumference $(\mathrm{cm})$ & $45 \pm 8$ & $56 \pm 5^{*}$ \\
Systolic blood pressure $(\mathrm{mmHg})$ & $128 \pm 10$ & $123 \pm 10$ \\
Diastolic blood pressure $(\mathrm{mmHg})$ & $86 \pm 12$ & $80 \pm 12$
\end{tabular}

${ }^{*} p<0.05$ 
to apply external pressure on the upper leg from an air-pressure source (compressor of $4.4 \mathrm{bar})$. The filling pressure was $7.5 \mathrm{kgf} / \mathrm{cm}^{2}$, the cuff was filled within $3 \mathrm{~s}$ and deflated within $0.3 \mathrm{~s}$, irrespectively of the occlusion pressure used. A mercury manometer was placed between the pressure tank and the cuff, in order to measure the pressure accurately.

The test started with a cuff-pressure $(\mathrm{Pc})$ of $70 \mathrm{mmHg}$, which will compress the venous system but leave the arterial system open. The effective pressure on the venous system $(\mathrm{Pv})$ can be estimated by 0.8 times Pc. ${ }^{9,13}$ After stabilisation of the congested limb, as verified by stabilisation of the plethysmographic record, the cuff was instantly deflated. If stabilisation was not achieved within $10 \mathrm{~min}$, the cuff was deflated and the measurement considered as not valid. The whole procedure was repeated with $\mathrm{Pc}$ of $60,50,40,30$ and $20 \mathrm{mmHg}$. When the pressure in the thigh veins is higher than $\mathrm{Pv}$ no volume changes in the calf will occur, which marks the end of the test. Each procedure of inflation and deflation (Fig 1) was followed by a $5 \mathrm{~min}$ rest period, used to calibrate the plethysmograph.

The brachial blood pressure was measured manually before the test was started
(Erkameter, Germany). During the test blood pressure and heart rate were measured continuously by means of the Finapres (2300 Finapres Blood Pressure Monitor, Ohmeda, USA) connected at the right middle finger which was kept at heart level.

\section{Curve analysis}

The curves show a gradual increase of the calf volume and the first part of the curve represents arterial inflow. The venous pressure distal to the cuff increases due to volume accumulation until the venous pressure reaches $\mathrm{Pv}$. At that time an equilibrium is achieved between inflow and outflow and no further volume increase occurs. The cuff is then instantly deflated and the limb volume returns to its initial value. The following parameters were calculated from the recordings of the plethysmograph during the test (Fig 1):

(1) Venous volume variation (VVV in \%: calculated as $\mathrm{dV} / \mathrm{V} \times 100 \%)$ is defined as the relative volume increase in the limb when a certain pressure has been applied and an equilibrium is established. The relationship between $\mathrm{Pv}$ and VVV is expressed in a diagram, the pressure volume (PV)-curve (Fig 2).

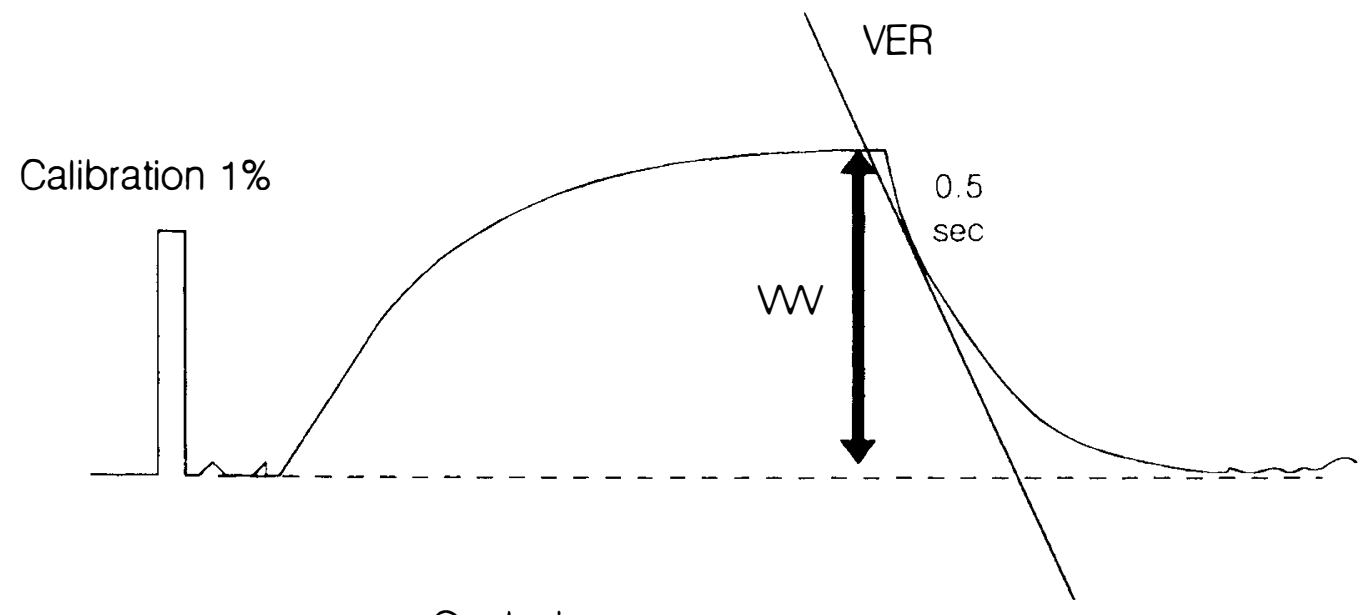

Occlusion pressure

Figure 1 Principle of venous occlusion plethysmography. VVV = venous volume variation (\%), $\mathrm{VER}_{0.5}=$ venous emptying rate at $0.5 \mathrm{~s}$ after deflation $(\mathrm{vol} \% / \mathrm{mmHg} / \mathrm{min})$. 


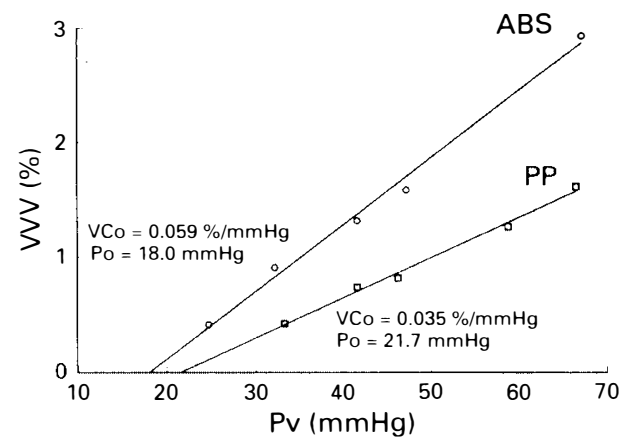

Figure 2 The pressure volume (PV)-curve for one representive individual with paraplegia (PP) and one representive able-bodied control subject $(\mathrm{ABS})$. VVV $=$ venous volume variation; $\mathrm{Pv}=$ occlusion pressure; $\mathrm{VCo}=$ venous capacity; Po $=$ resting venous pressure.

The intersection of this curve with the pressure-axis represents the venous pressure $(\mathrm{Po})$ in the resting thigh in a sitting position.

(2) Venous capacity ( $\mathrm{VCo}$ in $\mathrm{vol} \% / \mathrm{mmHg}$ ) at rest in the sitting position is characterised by the initial slope of the PVcurve.

(3) Venous emptying rate (VER in vol\%/ min) was calculated as the slope of the tangent at the curve $0.5 \mathrm{~s}$ after pressure release. The tangent at $0.5 \mathrm{~s}$ after deflation was chosen to avoid any cuff artefact in the volume decay curve. The $\mathrm{Pv}$ at $0.5 \mathrm{~s}$ after deflation $\left(\mathrm{Pv}_{0.5}\right)$ was calculated using the PV-curve. ${ }^{9}$ The relationship between $\mathrm{PV}_{0.5}$ and VER is expressed in a diagram, the pressure emptying (PE)-curve (Fig 3).

(4) Venous flow resistance (VFR in $\mathrm{mmHg}$ $\mathrm{min} / \mathrm{vol} \%$ ) is calculated, by analogy with Ohm's law, as 1 divided by the slope of the PE-curve (Fig 3).

The reproducibility of the methods was verified by testing $5 \mathrm{ABS}$ twice with 1 day in between.

\section{Statistical analysis}

Student's $t$-test was used to assess the significance of difference in VVV, VC, VER and VFR between PP and ABS. Pearson's correlation coefficient was calculated to establish correlations between VVV

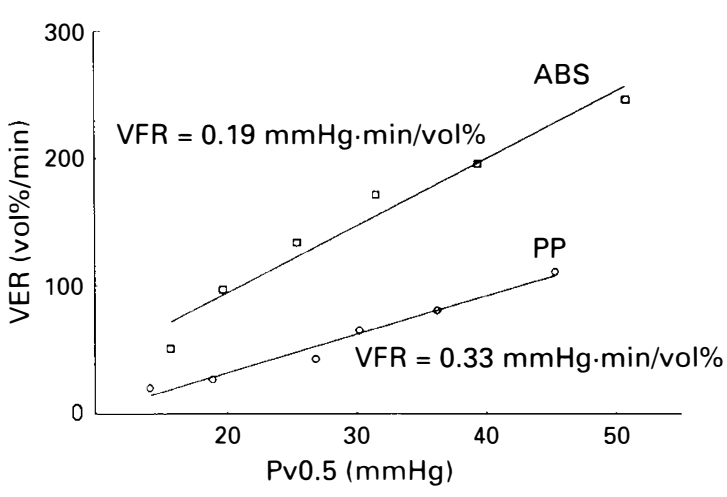

Figure 3 The pressure emptying (PE)-curve for individuals with paraplegia (PP) and ablebodied control subjects (ABS). VER = venous emptying rate after $0.5 \mathrm{~s}$ of deflation; $\mathrm{Pv}_{0.5}=$ occlusion pressure after $0.5 \mathrm{~s}$ of deflation; VFR = venous flow resistance.

and (i) the level of the lesion, (ii) the circumference of the calf and the thigh and (iii) the systolic and diastolic blood pressure. Statistical significance was accepted at $p<0.05$.

The reproducibility was expressed as a duplicate measurement error, calculated as: error $=\sqrt{ }\left(\sum \mathrm{d}^{2} / 2 n\right)(\mathrm{d}=$ difference between both measurements and $n=$ number of paired observations) and expressed as percentage of the indicated parameter.

\section{Results}

Age, body mass, height, heart rate and systolic and diastolic blood pressure were not significantly different between both groups. The circumferences of the calf and the thigh were significantly lower in PP than in ABS (Table I). The duplicated measurement error varied between 5 and $10 \%$ for all parameters calculated from the plethysmographic record during occlusion and deflation. Since VVV, VCo, VER as well as VFR were not significantly different between individuals with complete and incomplete lesions all PP were considered as being one group for further analyses (Table II).

VVV was significantly lower in PP than in ABS at any given occlusion pressure. Po, 
Table II The results of the venous occlusion plethysmography for individuals with paraplegia (PP) and able-bodied control subjects (ABS) as mean $\pm \mathrm{SD}$. VVV = venous volume variation at $\mathrm{Pc}$ of $50 \mathrm{mmHg}, \mathrm{VCo}=$ venous capacity at $\mathrm{Po}, \mathrm{Po}=$ resting venous pressure in the calf in sitting position.

\begin{tabular}{lcc}
\hline & PP & ABS \\
\hline VVV (\%) (at Pc: $50 \mathrm{mmHg})$ & $0.69 \pm 0.28^{*}$ & $1.37 \pm 0.38$ \\
VCo (vol\%/mmHg) & $0.029 \pm 0.010^{*}$ & $0.048 \pm 0.010$ \\
Po (mmH) & $24.4 \pm 7.8$ & $19.6 \pm 3.9$ \\
VFR (mmHg.min/vol\%) & $0.33^{*}$ & 0.19 \\
\hline
\end{tabular}

$* p<0.05$

however, did not differ significantly between both groups. VCo in PP was significantly lower compared to ABS (Figure 2, Table II).

PP demonstrated a significantly lower VER compared to ABS at any given pressure. VFR was significantly higher in PP than in ABS (Fig 3, Table III).

No significant correlations between VVV and either the level of the lesion, the circumference of the calf or thigh and the systolic or diastolic blood pressure could be observed, neither in PP nor in ABS.

\section{Discussion}

Strain gauge plethysmography is a simple, non-invasive and sensitive method to identify limbs with suspected venous incompetence. ${ }^{9,14-17}$ In this study the application of strain gauges with venous occlusion plethys- mography has been used in relation to individuals with paraplegia in order to examine the properties of the peripheral vascular system and to distinguish between two hypotheses, i.e. venous blood pooling versus venous atrophy. The reproducibility of the method used can be considered valid as indicated by the duplicate measurement error.

The vascular properties as found in this study for ABS were in agreement with previously reported values taking into consideration the difference in posture: sitting in the present study versus supine in previous studies. ${ }^{9-11.18}$ PP, however, demonstrated striking deviations in the parameters describing the properties of the venous vascular system in the lower limbs.

The low VVV in PP indicates a decrease in venous distensibility. ${ }^{9.17}$ This can be explained in two ways: the first explanation

Table III Results of venous outflow for individuals with paraplegia (PP) and able-bodied control subjects $(\mathrm{ABS})$ as mean $\pm \mathrm{SD}$

\begin{tabular}{|c|c|c|c|c|}
\hline \multirow[b]{2}{*}{$\mathrm{Pc}(\mathrm{mmHg})$} & \multicolumn{2}{|r|}{ PP } & \multicolumn{2}{|r|}{ ABS } \\
\hline & $\mathrm{Pv}(\mathrm{mmHg})$ & $\operatorname{VER}(\operatorname{vol} \% / \min )$ & $\mathrm{Pv}(\mathrm{mmHg})$ & $\operatorname{VER}(\operatorname{vol} \% / \mathrm{min})$ \\
\hline 20 & 14.1 & $19.8 \pm 5.8^{\mathrm{a}}$ & 15.7 & $50.4 \pm 18.0$ \\
\hline 30 & 18.9 & $26.4 \pm 8.7^{\mathrm{a}}$ & 19.7 & $96.6 \pm 34.0$ \\
\hline 40 & 26.8 & $42.6 \pm 12.1^{\mathrm{a}}$ & 25.4 & $133.8 \pm 47.4$ \\
\hline 50 & 30.2 & $64.8 \pm 17.6^{\mathrm{a}}$ & 31.5 & $171.6 \pm 62.8$ \\
\hline 60 & 36.2 & $80.4 \pm 27.0^{\mathrm{a}}$ & 39.4 & $196.2 \pm 71.7$ \\
\hline 70 & 45.3 & $111.0 \pm 30.4^{\mathrm{a}}$ & 50.8 & $246.4 \pm 84.8$ \\
\hline $\begin{array}{c}\mathrm{VFR}(\mathrm{mmHg} \\
\mathrm{min} / \mathrm{vol} \%)\end{array}$ & $0.33^{\mathrm{a}}$ & & 0.19 & \\
\hline
\end{tabular}

a $p<0.05$

$\mathrm{Pc}=$ cuff pressure; $\mathrm{Pv}=$ venous pressure at $0.5 \mathrm{~s}$ after deflation; VER = venous emptying rate at $0.5 \mathrm{~s}$ after deflation; VFR $=$ venous flow resistance. 
is related to the sympathetic denervation below the lesion and the inactivity of the leg muscle pump. Both of these factors may result in a continuously dilated venous system, which causes the so called 'venous blood pooling' in the legs. ${ }^{6,19-21}$ Consequently, the venous system will have less space left for blood to accumulate and VVV remains low. The second explanation is based on the muscle atrophy below the spinal cord lesion and the possibility of a concomitant vascular atrophy. This may result in a decrease of the venous vascular volume and, consequently, the space to accumulate blood and, thus, VVV will be limited.

Since Po did not differ significantly between each group the resting venous pressure in the calf of both groups can be considered equal. This indicates that Po is not influenced by a spinal cord injury. The Po values in this study were in the range of previously reported values after correction for posture differences. ${ }^{9,10}$

The lower VCo in PP may indicate an increased stiffness of both the venous walls and the tissue that surrounds the venous system in the lower limbs. This loss of distensibility may be the result of the continuous dilatation of the veins in 'venous blood pooling'. A decrease in the venous vascular volume related to the muscle atrophy may also lead to a loss of distensibility. In both situations, a given occlusion pressure will lead to a smaller transmural pressure in $\mathrm{PP}$ than in $\mathrm{ABS}$ and consequently VCo is lower.

The lower VER and concomitant higher VFR in PP compared to ABS indicates an enhanced venous outflow resistance in PP. These differences between PP and ABS are most probably based on differences in the total cross sectional area of the venous vessels. The remarkable differences in calf circumferences between PP and ABS are the result of an enormous muscle atrophy in the PP group. This muscle atrophy and the concomitant atrophy of the venous vascular bed below the lesion may explain the smaller total cross sectional area and, thus, the higher VFR in PP. In contrast, 'venous blood pooling' will not lead to a higher VFR. The results from this study therefore indicate that a 'venous blood pooling' in the legs of a paraplegic person is very unlikely.

Future research should be directed towards a further exploration of the consequences of these vascular changes on other circulatory problems such as decubitus. In addition, the question whether or not these vascular changes are reversible using functional neuromuscular stimulation of the paralysed lower limb muscles should be addressed.

In conclusion: this study demonstrates that the vascular properties in the lower limbs of individuals with long-standing paraplegia are different from those in ablebodied control subjects. The reduction in leg volume in combination with an enhanced venous outflow resistance indicates a reduction in the cross sectional area of the veins in PP. This supports the hypothesis of venous atrophy as an adaptation to inactivity and muscle atrophy rather than the effect of venous blood pooling caused by sympathetic denervation or muscle pump inactivity.

\section{Acknowledgement}

Special thanks are exerted to Dr A J M Brakkee for his helpful advice, to Dr M A van't Hof for statistical help and to Mr A C A Visser for technical assistance.

\section{References}

1 Mathias CJ (1988) Cardiovascular control in spinal man. Ann Rev Physiol 50: 577-592.

2 Hopman MTE (1994) Circulatory responses during arm exercise in individuals with paraplegia. Int J Sports Med 15: 126-131.

3 Rothe CF (1983) Reflex control of veins and vascular capacitance. Physiol Rev 63: 1281-1342.

4 Rowell LB (1984) Reflex control of regional circulation in man. J Auton Nerv Syst 11: 101-114.

5 Bidart Y, Maury M (1973) The circulatory behavior in complete chronic paraplegia. Paraplegia 11: 1-24.

6 Kinzer SM, Convertino VA (1989) Role of leg vasculature in the cardiovascular response to arm work in wheelchair dependent populations. Clin Physiol 9: 525-533. 
7 Hopman MTE, Verheijen PHE, Binkhorst RA (1993) Volume changes in the legs of paraplegic subjects during arm exercise. J Appl Physiol 75: 2079-2083.

8 Waters RL, Adkins RH, Yakura JS (1991) Definition of complete spinal cord injury. Paraplegia 29: 573-581.

9 Brakkee AJM, Kuiper JP (1982) Plethysmographic measurement of venous flow resistance in man. Vasa 2: 166-173.

10 Pointel JP, Gin H, Drouin P, Vernhes G, Debry G (1981) Venous plethysmography: measuring techniques and normal values. Angiology 32: 145-154.

11 Fernandes e Fernandes J, Horner J, Needham T, Nicolaides A (1979) Ambulatory calf volume plethysmography in the assessment of venous insufficiency. Br J Surg 66: 327-330.

12 Brakkee AJM, Vendrik AJH (1966) Strain-gauge plethysmography; theoretical and practical notes on a new design. J Appl Physiol 21: 701-704.

13 Thulesius O (1973) Capillary filtration under normal and pathological conditions. Angiologia 10: $198-213$.

14 Whitney RJ (1953) The measurement of volume changes in human limbs. J Physiol London 121: 1-27.

15 Walker RL, MacKay IFS, Van Loan P (1967) Vascular responses to venous congestion. J Appl Physiol 22: 889-899.

16 Barendsen GJ, Van den Berg J (1976) Venous pressure volume relation and calf blood flow determined by changes in posture. Cardiovasc Res 10: 206-213.

17 Cheah LH, Parkin A, Gilson P, Elliot S, Hall AJ (1989) Limb volume measurements in peripheral arterial disease. Clin Phys Physiol Meas 10: 75-79.

18 Struckman J, Floistrup Vissing S, Hjortso E (1992) Ambulatory strain-gauge plethysmography and blood volume scintimetry for quantitative assessment of venous insufficiency. Clin Physiol 12: 277-285.

19 Hjeltnes $N$ (1977) Oxygen uptake and cardiac output in graded arm exercise in paraplegics with low level spinal lesions. Scand J Rehabil Med 9: 107-113.

20 Davis GM, Shephard RJ (1988) Cardiorespiratory fitness in highly-active versus inactive paraplegics. Med Sci Sports Exerc 20: 463-468.

21 Hopman MTE, Oeseburg B. Binkhorst RA (1992) Cardiovascular responses in paraplegic subject during arm exercise. Eur J Appl Physiol 65: 73-78. 